\title{
Multiple Marker Screening for Down Syndrome- Whom Should We Screen?
}

\author{
Sara Cate, $M D, M P H$, and Susie Ball, MS, CGC
}

Background: The multiple marker test highlights the complexities of an expanding number of genetic tests that family physicians can offer to their patients. Many concerns surround the use of the multiple marker test, including a poor understanding of the test by physicians and patients, limited sensitivity, increased anxiety among women, and the risks of amniocentesis in patients who have false-positive results.

Methods: An online search of the medical literature was used to select English-language articles addressing the burden of suffering from Down syndrome, and efficacy, cost-effectiveness, and psychological effects of screening.

Results and Conclusions: Down syndrome occurs in 0.99 per 1000 births, and the risk increases with age. Between $10 \%$ and $20 \%$ of infants die in the first year, predominantly from heart defects. Special education, the move away from institutionalization, and more employment opportunities have improved the outlook for these persons. The multiple marker test has an overall detection rate for Down syndrome of $56 \%$ with a $7 \%$ false-positive rate. A laboratory cutoff ratio of $1: 190$ is the most efficient for optimizing the detection rate and minimizing the false-positive rate. Limited screening to women older than 30 years appears most cost-effective, reducing losses of normal fetuses secondary to amniocentesis and the number of pregnant patients requiring pretest counseling. The family physician needs to inform the patient of the nature, purpose, and risks of screening including the psychological effects. Pretest counseling should be nondirective. (J Am Board Fam Pract 1999;12:367-74.)

With the introduction of a screening test for Down syndrome, genetic screening has been broadened to include women who have not had access to it in the past. Many family physicians are offering pregnant women multiple marker testing for Down syndrome between $15 \frac{1}{2}$ and 20 week's gestation. Maternal age and levels of two or more serum analytes (maternal serum $\alpha$-fetoprotein [MSAFP], human chorionic gonadotropin [hCG], and unconjugated estriol [uE3]) are used to calculate a patient-specific risk. This risk estimate is then compared with a selected age-related risk for Down syndrome. If this patient's specific risk is equal to or greater than the age-related risk, the test is positive.

Before the introduction of serum marker testing, most Down syndrome infants were not detected before birth. Screening was based on the increasing risk of Down syndrome with maternal age. Women

Submitted, revised 22 January 1999.

From the Central Washington Family Medicine Residency Program (SC), and the Central Washington Genetics Program of the Yakima Valley Memorial Hospital (SB), Yakima. Address reprint requests to Sara Cate, MD, MPII, Central Washington Family Medicine Residency Program, 1806 West Lincoln Ave, Yakima, WA 98902. who would be 35 years or older at the time of delivery were offered amniocentesis at 16 to 18 weeks' gestation. ${ }^{1}$ Many women chose not to have amniocentesis. In addition, $80 \%$ of most Down syndrome infants were born to women younger than 35 years. The infants of these women were at lower risk for Down syndrome, but the mothers accounted for a greater proportion of births. Because of these factors, even if the rate of amniocentesis were as high as $50 \%$ in women older than 35 years, the reduction in the incidence of Down syndrome would be only $10 \%{ }^{2}$

In 1984 Merkatz et $\mathrm{al}^{3}$ found that MSAFP concentrations were on average lower in pregnancies with fetal autosomal trisomy. Subsequently, a large clinical trial found that MSAFP as a screening test in the second trimester could detect $25 \%$ of cases of Down syndrome in pregnant women younger than 35 years. $^{4}$

This finding spurred a search for other serum analytes that could increase the predictive accuracy of screening. Pregnant mothers carrying fetuses affected with Down syndrome were sometimes found to have higher levels of hCG and lower than normal levels of uE3. 5,6 A large clinical trial of 
women aged 16 to 41 years showed that measuring all three of these maternal serum analytes, MSAFP, hCG and uE3, increased the detection of Down syndrome to approximately $60 \%{ }^{7}$

After several years of experience with the multiple marker test, concerns are being raised about its use. ${ }^{8}$ Many patients and physicians have a poor understanding of the test. There is also concern about its limited sensitivity, the number of women with false-positive test results who experience increased anxiety and are exposed to the risks of amniocentesis, and the number who receive positive results but do not have follow-up testing. The purposes of this article are to review the burden of suffering from Down syndrome, the efficacy and cost-effectiveness of serum screening, and the psychologic effects on the family of proceeding with the screening test or diagnostic confirmation, as well as to recommend strategies to enhance family physicians' appropriate use of the test and counseling of patients.

\section{Methods}

The Internet was used to conduct an online search of the medical literature, using the key words "multiple marker screening," "prenatal diagnosis," and "Down syndrome," to select English-language articles addressing the burden of suffering from Down syndrome and to assess the efficacy, costeffectiveness, and psychological effects of screening.

\section{Burden of Suffering from Down Syndrome}

The incidence of Down syndrome is 0.99 ( 0.85 to 1.43) per 1000 births (live and still births). ${ }^{2,9}$ This rate also includes cases of Down syndrome diagnosed during the first year of life. The prevalence of Down syndrome is 0.9 per 1000 live births. The rate of Down syndrome increases with maternal age. A 20-year-old woman's risk of having a child with Down syndrome is 0.6 per 1000 live births, a 30 -year-old woman's risk is 1.1 , a 35 -year-old woman's risk is 2.65 , and a 45 -year-old woman's risk is 33.0. ${ }^{1}$ Down syndrome is more common than neural tube defects $(0.36$ to 0.46 per 1000 live births), which are screened for prenatally. ${ }^{10}$ It is the most common of the trisomy syndromes and is caused by a critical portion of chromosome 21 occurring three times instead of twice in some or all cells. The syndrome includes hypotonia, varying degrees of mental and growth retardation, heart defects, and an increased risk of leukemia and Alzheimer disease.

The period of highest mortality risk is in infancy. Between $10 \%$ and $20 \%$ of infants with Down syndrome die during the first year of life. ${ }^{11,12}$ Thirty percent to $40 \%$ of infants with Down syndrome have a heart defect at birth. The predominant causes of death in the first year are congenital heart disease, other malformations, and infection. Of those children who have congenital heart defects, $72 \%$ survive to the age of 1 year and $45 \%$ to the age of 6 years. Survival at 1 year is improved considerably for those children whose heart defects are treated surgically rather than medically. In children who do not have heart disease, the probabilities of survival are $93 \%$ and $88 \%$ at 1 and 6 years, respectively. ${ }^{12}$

Most persons with Down syndrome have mild to moderate mental retardation (IQ 30 to 60 ). ${ }^{13}$ Persons with Down syndrome at the upper end of the IQ range might attain 4th to 6 th grade reading skills, can provide for basic self-help needs, and have varying degrees of educational achievement and social and occupational skills. They need special education, training facilities, and frequently sheltered living and work situations. ${ }^{14}$ Persons with Down syndrome have a shortened lifespan of approximately 68 years. ${ }^{15}$

Studies regarding the impact of a child with Down syndrome on the family report both positive and negative effects. Parents devote more time to child care and spend less time in social activities. ${ }^{16}$ Siblings of Down syndrome children have more behavior problems and mothers more depression. ${ }^{17,18}$ When compared with families with no disabled children, families with Down syndrome children show no difference in individual, family, and marital functioning. ${ }^{19}$ Children and youth with disabilities can make positive contributions to the family. Siblings of persons with disabilities show greater tolerance, compassion, and awareness of prejudice and its consequences. ${ }^{20}$ This sometimescontradictory information might reflect that caring for a Down syndrome child has its ups and downs. Furthermore, there are many factors, such as availability of support services and financial and family cohesiveness, that influence outcomes in studies examining the impact of a handicapped child on the family. ${ }^{21}$

The lifetime economic costs of Down syndrome have been estimated to be $\$ 410,000$ per case of 
Down syndrome (1988). The lifetime costs for each case of Down syndrome were among the highest when compared with other birth defects. ${ }^{22}$

Parent advocacy groups and an increased understanding of the ultimate potential of persons with Down syndrome has led to their greater integration into society. The outlook for a child with Down syndrome has improved substantially during the past 20 years as a result of improved support services such as special education, the move away from institutionalization, and expanded employment opportunities. $^{23}$

\section{Efficacy of Screening}

The sensitivity and false-positive rate (100 - specificity) are important for assessing the efficacy of a screening test. ${ }^{24}$ Generally, a good screening test should have a sensitivity rate in the range of $80 \%$ to $90 \%$ so that the test does not miss a large number of cases. The false-positive rate is important in screening for Down syndrome if exposure to the risks of amniocenteses by pregnant women carrying normal fetuses is to be limited. Generally this rate should be less than $10 \%$.

The positive predictive value and the negative predictive value are useful when counseling about the probability of the disorder when there is a positive or negative test result. The positive predictive value and negative predictive value are affected by the prevalence of the disease. The negative predictive value is higher the lower the prevalence of the disease in the population, and the positive predictive value is higher the greater the prevalence of the disease.

Table 1 shows the sensitivity, false-positive rate (100 - specificity), and positive and negative predictive values of the triple marker test for two different cutoffs in a study of 16,067 women. This study is one of the first to measure the effectiveness of screening in a large population, and to compare the efficacy of screening with two different cutoffs. Overall, the positive predictive value of the multiple marker test is low. The test is much better at excluding the possibility of Down syndrome, as indicated by the very high negative predictive value. ${ }^{7}$

The laboratory choice of a cutoff level determines what is a positive result. Most laboratories use a risk ratio of $1: 270$ as their cutoff point. This cutoff level is the second trimester risk of Down syndrome in a 35 -year-old pregnant woman. ${ }^{25}$ This
Table 1. Numbers of Positive Screening Results, Amniocenteses, Cases of Down Syndrome, Sensitivity, Percent-False Positive (100 Minus Specificity) and Predictive Values Using Two Different Cutoffs.

\begin{tabular}{lcc}
\hline & \multicolumn{2}{c}{$\begin{array}{c}\text { Second-Trimester } \\
\text { Cutoff }\end{array}$} \\
\cline { 2 - 3 } Variables & $1: 190$ & $1: 270$ \\
\hline Positive results (No.) & 1141 & 1508 \\
Amniocenteses* (No.) & 533 & 725 \\
Cases detected (No.) & 13 & 14 \\
Sensitivity (\%) & 56 & 60 \\
False positive (\%) & 7 & 9 \\
Positive predictive value (\%) & 1.1 & 0.9 \\
Negative predictive value (\%) & 99.9 & 99.9 \\
\hline
\end{tabular}

Adapted from Haddow et al. ${ }^{7}$

Note: values based on data for 16,067 women screened with an average age of 27.2 years; 23.4 second trimester cases of Down syndrome expected.

*Six hundred eight women excluded after verification of gestational age or refusing amniocentesis $(82 \%$ of women offered amniocentesis agreed to procedure).

${ }^{\dagger}$ Numbers include cases diagnosed by amniocentesis and at delivery (those who did not undergo amniocentesis).

level of risk was chosen because it is approximately the same as that for a miscarriage from amniocentesis. Some laboratories use the higher second trimester risk ratio of $1: 190$ as a cutoff point, which is the level of risk for Down syndrome in a 36- to 37-year-old pregnant woman.

The choice of a different high-risk cutoff point will affect the sensitivity and false-positive rate. By decreasing the cutoff point to $1: 270$, the sensitivity increases, but there are more false-positive results (Table 1). An increase in the sensitivity of the test will result in more pregnant women having falsepositive findings and thus being exposed to the risks of amniocenteses. A recent meta-analysis compared sensitivity and false-positive rates for different cutoff points using receiver operator curves. A cutoff point of 1:190 was the most efficient for predicting Down syndrome for all age groups of pregnant women while minimizing the false-positive rate. ${ }^{26}$

In addition to choice of high-risk cutoff point, the sensitivity and predictive value of the multiple marker test varies depending on the age of the screened population. Table 2 shows that the sensitivity of the test increases with age, but so does the false-positive rate, which increases to $70 \%$ in 44 year-old women. The positive predictive value of the test also increases with age, as does the prevalence of Down syndrome. ${ }^{27}$ 
Table 2. Age-Related Sensitivity, False-Positive Rate, and Positive Predictive Value for Women Aged 16 to 44 Years, Using a Risk Cutoff of 1:300.

\begin{tabular}{lccc}
\hline $\begin{array}{l}\text { Age } \\
\text { (years) }\end{array}$ & Sensitivity & $\begin{array}{c}\text { False-Positive } \\
\text { Rate }\end{array}$ & $\begin{array}{c}\text { Positive Predictive } \\
\text { Value }\end{array}$ \\
\hline 16 & 44.3 & 3.1 & 0.9 \\
18 & 44.3 & 3.1 & 0.9 \\
20 & 44.7 & 3.2 & 0.9 \\
22 & 45.2 & 3.2 & 0.9 \\
24 & 46.5 & 3.6 & 0.9 \\
26 & 46.5 & 4.1 & 0.9 \\
28 & 51.6 & 4.7 & 1.0 \\
30 & 56.0 & 6.1 & 1.0 \\
32 & 62.0 & 8.7 & 1.0 \\
34 & 69.5 & 12.5 & 1.2 \\
36 & 78.0 & 19.0 & 1.3 \\
38 & 85.5 & 28.6 & 1.6 \\
40 & 91.6 & 40.9 & 2.0 \\
42 & 95.7 & 55.3 & 2.6 \\
44 & 98.1 & 70.0 & 3.8 \\
\hline
\end{tabular}

Adapted from Reynolds et al. ${ }^{27}$ Computer simulated percentages based on published values for distributions of analytes in Down syndrome screening.

The rate of women obtaining amniocenteses might vary among communities, which will affect the prenatal detection of Down syndrome. The sensitivity of the screening test might be adequate, but if the rate of patients undergoing the diagnostic test is low, the effectiveness of the screening program will be compromised.

In summary, the sensitivity and false-positive rates of the multiple marker test can vary depend- ing on specific characteristics of the patient community and local laboratory. With a cutoff point of $1: 190$, the overall sensitivity for the multiple marker test is approximately $56 \%$ and the falsepositive rate is $7 \%$. The negative predictive value of the test is $99.9 \%$. Sensitivity and false-positive rates will vary depending on the age structure of the population. If the average age of the screening population is very young, the sensitivity of the screening program will be less than if the average age is older. The sensitivity of the screening test improves in older age groups, but this improvement is accompanied by an increase in the falsepositives rate. A cutoff point of 1:190 is the most efficient for maximizing sensitivity while minimizing the false-positive rate. The family physician can encourage a multiple marker screening policy that minimizes false-positive results and maximizes the sensitivity of the test.

\section{Costs of Screening}

Widespread adoption of the multiple marker test has preceded a full discussion on how the various factors affecting the detection rate might make this screening test more or less worthwhile. Decision analysis is one way to examine the costs of screening by factoring in such variables as age-related differences in sensitivity and specificity, rates of uptake of amniocentesis, and different cutoff points for a positive test. ${ }^{28}$

A selected number of screening options is displayed in Table 3. Offering screening to all women

Table 3. Results of Decision Analysis of the Multiple Marker Screening Test for Four Screening Strategies-Number of Predicted Events per Year.

\begin{tabular}{|c|c|c|c|c|c|}
\hline Program & $\begin{array}{l}\text { Women } \\
\text { Offered } \\
\text { Screening } \\
\text { (No.) }\end{array}$ & $\begin{array}{l}\text { Amniocenteses } \\
\text { (No.) }\end{array}$ & $\begin{array}{l}\text { Miscarriages From } \\
\text { Amniocenteses per } \\
\text { Case of Down } \\
\text { Syndrome Detected } \\
\text { (No.) }\end{array}$ & $\begin{array}{l}\text { Down Syndrome } \\
\text { Cases Detected } \\
\text { (No.) }\end{array}$ & $\begin{array}{l}\text { Cost per Case of Down } \\
\text { Syndrome Detected } \\
(\$)\end{array}$ \\
\hline $\begin{array}{l}\text { Serum testing offered to all } \\
\text { pregnant women; high-risk } \\
\text { test result }>1: 250\end{array}$ & 7533 & 290 & 0.45 & 6.4 & 42,500 \\
\hline $\begin{array}{l}\text { Serum testing offered to all } \\
\text { pregnant women; high-risk } \\
\text { test result }>1: 100\end{array}$ & 7533 & 100 & 0.20 & 4.9 & 37,400 \\
\hline $\begin{array}{l}\text { Women }>35 \text { years offered } \\
\text { amniocentesis }\end{array}$ & 874 & 660 & 1.40 & 4.7 & 61,200 \\
\hline $\begin{array}{l}\text { Serum testing offered to } \\
\text { pregnant women }>30 \text { years; } \\
\text { high-risk test result }>1: 250\end{array}$ & 3066 & 120 & 0.25 & 4.7 & 25,500 \\
\hline
\end{tabular}

Adapted from Fletcher et al. ${ }^{28}$ 
using a cutoff of greater than 1:250 for a high-risk result detects most cases of Down syndrome. The trade-off is an increased number of false-positive results, leading to more amniocenteses and more miscarriages of potentially normal fetuses (approximately 1 for every 2 Down syndrome infants), and a higher cost. Testing all women but increasing the high-risk result cutoff point from 1:250 to 1:100 yields fewer false-positive results and is a lower cost option. Although this option has a lower rate of miscarriage of normal fetuses, it selects for fewer Down syndrome cases. The lowest cost option is screening only women older than 30 years. This option minimizes the number of women who are offered and need pretest counseling.

Offering amniocenteses to women older than 35 years is the most expensive method and has the highest rate of miscarriage per case detected. Despite the high false-positive rate in older women, there has been support for offering serum screening first in women 35 years or older. Haddow et al and others ${ }^{29,30}$ estimate that restricting amniocenteses to women with a second-trimester risk of Down syndrome greater than 1 in 200 (the risk of amniocentesis-related miscarriage in their study) could save $\$ 250$ million a year and avoid the loss of 14,000 fetuses unaffected by trisomy 21 . On the other hand, women who had negative test results would give birth to 320 additional Down syndrome children. A further rationale for offering the multiple marker screening first to women 35 years or older is that a positive result might encourage those ambivalent about diagnostic testing to have an amniocentesis. This decision would also theoretically lead to the detection of more fetuses with Down syndrome

This decision analysis provides a glimpse of the big picture-there are costs and risks of different screening options, including the loss of normal fetuses. This analysis suggests that serum screening of pregnant women older than 30 years is most cost-effective, resulting in fewer normal fetuses lost. The application of such a policy might be difficult in the United States because such factors as equity and medicolegal considerations might override a more cost-effective screening strategy. ${ }^{31}$

\section{Clinical Guidelines}

The US Preventive Services Task Force (USPSTF) and the Canadian Task Force on the Periodic Health Examination have completed comprehen- sive reviews of the literature. Both support multiple marker screening for Down syndrome for women who have adequate access to counseling, interpretation, and follow-up. ${ }^{32,33}$ The Canadian Task Force supports the multiple marker test for women younger than 35 years, and the USPSTF supports the multiple marker test for all women. The USPSTF recommends it, but gives it a $\mathrm{B}$ recommendation.

The USPSTF defines counseling as providing information on the screening test itself, the likelihood of needing confirmation with amniocentesis, and associated risks. Moreover, they recommend discussing with the patient the potential outcomes associated with giving birth to a child with Down syndrome or of aborting a Down syndrome fetus. The Canadian Task Force raises concerns about the limited sensitivity of the multiple marker test, the number of false-positive test results, and the number of women who receive positive results but do not have follow-up diagnostic testing. They suggest that these limitations weigh most heavily on the obstetric care provider.

\section{Patient and Physician Understanding of the Test}

Both patients and physicians find the multiple marker test difficult to understand. Women understand the practical aspects of undergoing the test but poorly understand what the test detects and the implications of a positive or negative test. ${ }^{34}$ The reasons are probably multifactorial. Low educational level, youth, and non-English-speaking are barriers to understanding the test. ${ }^{35}$ Moreover, the physician might not have the resources to provide adequate counseling. ${ }^{36}$ Research also suggests that obstetric providers' understanding of the performance (sensitivity, specificity, and positive predictive value) and interpretation of serum screening is poor. Providers complain that the test is too complex to explain and understand. 8,37

Lack of understanding of the test could contribute to increased anxiety for women after a positive result from serum screening. ${ }^{38,39}$ One reason is that patients are unfamiliar with the difference between a screening and a diagnostic test. Lack of definitive information is stressful. Women who choose not to have an amniocentesis are significantly more anxious than those who do. ${ }^{40} \mathrm{~A}$ physician might also be at risk for being sued if a patient feels that she did not receive adequate information about the results of the test. ${ }^{41}$ 


\section{Pretest Counseling}

The family physician's best chance of avoiding these adverse consequences is by discussing the nature, purpose, and risks of screening with the patient. This discussion should include possible psychological effects of the screening test and amniocentesis, the positive and negative attributes of Down syndrome, and the patient's feelings about abortion. The family physician might be better able to provide this counseling than a genetic counselor because of experience with and knowledge of the family, but the physician must be careful not to be directive. The goal of genetic counseling is nondirective education for which genetic counselors have specific training.

In a survey of physicians on how they would counsel a couple about prenatal diagnosis of a genetic disorder, one half of the physicians felt comfortable counseling and one half did not and would refer. Family physicians were more likely than obstetricians to discuss a prenatal diagnosis with a patient rather than refer her to a specialist. On the other hand, when compared with other specialists, family physicians and internists were more likely to give their opinions about abortion. Men were more likely than women to be directive and deviate from the tradition of nondirectiveness. ${ }^{42}$ Physicians or genetic counselors might express their opinion directly or in a covert manner by focusing attention on prenatal risks and the consequences of genetic disease. For example, directive counseling can occur when the physician focuses on the negative or positive side of Down syndrome, influencing a patient's choice of abortion. ${ }^{43} \mathrm{It}$ is important in nondirective counseling that the physician recognize how his or her feelings about abortion or Down syndrome might influence a patient's decision making. ${ }^{44}$ If a bias does exist, referral to a genetic counselor for pretest counseling is warranted.

Comprehensive discussion of genetic counseling is beyond the scope of this article, but the following are ingredients to successful pretest counseling and avoiding the adverse psychological consequences for patients. Before the visit the physician should prepare by reviewing the disorder screened, the performance of the screening test, and other pertinent medical and genetic data. Using local or regional genetic counseling services can help bridge the knowledge gap for the family physician. A genetic counselor might be able to provide a workshop on genetic screening. Regional genetics networks might also have written patient and physician information on the multiple marker screening test. ${ }^{45}$ The screening information should include material geared to the reading level of the physician's patient population. Anxiety regarding the test has been shown to decrease and knowledge improve when patients are given written information about the multiple marker screening test. ${ }^{46}$

During the visit the physician should ascertain the patient's resources, values, expectations, and knowledge level. The counselor's job is to facilitate the process of patient decision making. The physician should elicit any questions about the test and be attentive to the emotional state of the patient. The physician can begin by describing that the goal of screening is to detect Down syndrome before birth and can then review with the patient what she knows about Down syndrome and clarify any misconceptions. ${ }^{47}$ The patient needs to know the screening test is not a diagnostic test but a blood test that can predict her chances of carrying a Down syndrome infant. Only amniocentesis can determine whether her baby does or does not have this disorder. The multiple marker test is excellent at ruling out Down syndrome (eg, if the test result is negative, it is likely to be a true negative). Its average detection rate is $56 \%$, and the false-positive rate is $7 \%$.

The physician should ask the patient whether she would obtain an amniocentesis to confirm a positive test. She needs to be informed that there is a chance ( $1 \%$ or less) of miscarriage related to the amniocentesis. Those who strongly state that under no circumstances would they undergo amniocentesis might prefer to decline testing. If the patient desires testing but is not sure about amniocentesis, she might experience increased anxiety during the remainder of the pregnancy if she has a positive test result but decides not to have amniocentesis. After counseling a pregnant woman, a questionnaire to assess knowledge about maternal screening will help determine what information might not have been understood. ${ }^{48} \mathrm{~A}$ patient can be offered a follow-up appointment to discuss test results and to help alleviate any anxiety she might feel, particularly after a positive test.

The first step after a positive test result is to review the information (maternal weight, age, race, the presence of insulin-dependent diabetes mellitus, and gestational age) submitted to the labora- 
tory with the blood sample. If this information is accurate, the next step is to order a sonogram to verify dates of conception and confinement. If a sonogram has been done, and the estimated date of confinement is within 2 weeks of the date based on the last menstrual period, the sonogram should not be repeated.

In posttest counseling, a positive test result can be put into perspective for the patient immediately. For example, a 30-year-old woman with a positive test result has a $1 \%$ (or 1 in 100) chance that it was a true positive, whereas a 44 -year-old woman with a positive test result has a $3.8 \%$ (or 1 in 26) chance that it was a true positive (Table 2). Most laboratories provide a patient risk estimate rather than just reporting whether the test result is positive or negative. This more accurate risk estimate should be used in counseling the patient. All patients receiving a positive test result should be referred to a genetic counselor.

\section{Conclusions}

The greater role of genetic screening in prenatal care has not been risk-free; not only has it increased the burden of pretest counseling on the primary care provider, it has also exposed more women to the risks of amniocentesis and false-positive results. At the same time, there has been an appropriate change in the role of prenatal diagnosis from eugenics to provision of information. Whether the patient uses that information for abortion or emotional preparation and organization of resources for a Down syndrome infant is the patient's choice. Given the differing values about the acceptability of Down syndrome, the family physician, with a good understanding of the test, can enhance a patient and her family's ability to make an informed decision about screening.

There is, however, a tension between the individual's needs and societal costs (eg, loss of normal fetuses). These costs can be minimized (1) by using a higher multiple marker test cutoff ratio $(1: 190)$ to limit false-positive results, (2) by paying attention to provider miscarriage rates secondary to amniocentesis, (3) by focusing screening on higher risk groups (eg, women older than 30 years), (4) by selecting through pretest counseling a group of women who understand and accept the risks and benefits of screening, and (5) by continuing the search for serum analytes that improve the sensitivity and specificity of serum screening.

\section{References}

1. I Iook EB. Rates of chromosome abnormalities at different maternal ages. Obstet Gynecol 1981;58:282-5.

2. Adams MM, Erickson JD, Layde PM, Oakley GP. Down's syndrome. Recent trends in the United States. JAMA 1981;246:758-60.

3. Merkatz IR, Nitowsky HM, Macri JN, Johnson WE. An association between low maternal serum alphafetoprotein and fetal chromosomal abnormalities. Am J Obstet Gynecol 1984;148:886-94.

4. Combining maternal serum alpha-fetoprotein measurements and age to screen for Down syndrome in pregnant women under age 35. New England Regional Genetics Group Prenatal Collaborative Study of Down Syndrome Screening. Am J Obstet Gynecol 1989;160:575-81.

5. Bogart MH, Pandian MR, Jones OW. Abnormal maternal serum chorionic gonadotropin levels in pregnancies with fetal chromosome abnormalities. Prenat Diagn 1987;7:623-30.

6. Canick JA, Knight GJ, Palomaki GE, Iaddow JE, Cuckle HS, Wald NJ. Low second trimester maternal serum unconjugated oestriol in pregnancies with Down's syndrome. Br J Obstet Gynaecol 1988;95: 330-3.

7. Haddow JE, Palomaki GE, Knight GJ, et al. Prenatal screening for Down's syndrome with use of maternal serum markers. N Engl J Med 1992;327:588-93.

8. Carroll JC, Reid AJ, Woodward CA, et al. Ontario Maternal Serum Screening Program: practices, knowledge and opinions of health care providers. CMAJ 1997;156:775-84.

9. Centers for Disease Control and Prevention. Down syndrome prevalence at birth-United States, 19831990. MMWR Morbid Mortal Wkly Rep 1994;43: 617-22.

10. US Preventive Services Task Force. Screening for neural tube defects. In: Guide to clinical preventive services. Report of the US Preventive Services Task Force Baltimore, Md: Williams \& Wilkins, 1996: 467-483.

11. Brookes ME, Alberman E. Early mortality and morbidity in children with Down's syndrome diagnosed in two regional health authorities in $1989 . \mathrm{J} \mathrm{Med}$ Screen 1996;3:7-11.

12. Mikkelsen MA, Poulsen II and Nielsen KM. Incidence, survival and mortality in Down Syndrome in Denmark. A J Med Genet Suppl 1990;7:75-8.

13. Gorlin RJ, Cohen MM, Leven LS. Syndromes of the head and neck. 3rd ed. New York: Oxford University Press, 1990:393.

14. Earl DT, Blackwelder RB. Management of chronic medical conditions in children and adolescents. Prim Care 1998;25:253-70.

15. Baird PA, Sadovnick AD. Life tables for Down syndrome. Hum Genet 1989;82:291-2.

16. Barnett WS, Boyce GC. Effects of children with 
Down syndrome on parents' activities. Am J Ment Retard 1995;100:115-27.

17. Cuskelly M, Gunn P. Maternal reports of behavior of siblings of children with Down syndrome. Am J Ment Retard 1993;97:521-9.

18. Scott BS, Atkinson, L, Minton HL, Bowman T. Psychological distress of parents of infants with Down syndrome. Am J Ment Retard 1997;102:161-71.

19. Riper MV, Ryff C, Pridham K. Parental and family well-being in families of children with Down syndrome: a comparative study. Res Nurs Health 1992; 15:227-35.

20. Elkins TE, Brown D. The cost of choice: a price too high in the triple screen for Down syndrome. Clin Obstet Gynecol 1993;36:532-40.

21. Murphy MA. The family with a handicapped child: a review of the literature. J Dev Behav Pediat 1982;3: 73-82.

22. Waitzman NJ, Romano PS, Scheffler RM. Estimates of the economic costs of birth defects. Inquiry 1994; 31:188-205.

23. Haslam RH, Milner R. The physician and Down syndrome: are attitudes changing? J Child Neurol 1992;7:304-10.

24. Lilienfeld AM, Lilienfeld DE Foundations of epidemiology. 2nd ed. New York: Oxford University Press, 1980:151.

25. Palomaki GE, Knight GJ, McCarthy J, Haddow JE, Eckfeldt JH. Maternal serum screening for fetal Down syndrome in the United States: a 1992 survey. Am J Obstet Gynecol 1993;169:1558-62.

26. Conde-Agudelo A, Kafury-Goeta AC. Triplemarker test as screening for Down syndrome: a meta-analysis. Obstet Gynecol Surv 1998; 53:369-76.

27. Reynolds TM, Nix AB, Dunstan FD, Dawson AJ. Age-specific detection and false-positive rates: an aid to counseling in Down syndrome risk screening. Obstet Gynecol 1993;81:447-50.

28. Fletcher J, Hicks NR, Kay JD, Boyd PA. Using decision analysis to compare policies for antenatal screening for Down's syndrome. BMJ 1995; 311:351-6.

29. Haddow JE, Palomaki GE, Knight GJ, Cunningham GC, Lustig LS, Boyd PA. Reducing the need for amniocentesis in women 35 years of age or older with serum markers for screening. N Engl J Med 1994;330:1114-8.

30. Pauker SP, Pauker SG. Prenatal diagnosis - why is 35 a magic number? $N$ Engl J Med 1994; 330:1151-2.

31. Ubel PA, DeKay ML, Baron J, Asch DA. Cost-effectiveness analysis in a setting of budget constraints-is it equitable? N Engl J Med 1996;334:1174-7.

32. Screening for Down syndrome. In: Guide to clinical preventive services: report of the US Preventive Services Task Force. Baltimore: Williams \& Wilkins, 1996:449-65.
33. Dick PT, Canadian Task Force on the Periodic $\stackrel{C}{\supset}$ Health Examination. Periodic health examination, $\frac{}{3}$ 1996 update: 1. Prenatal screening for and diagnosis o of Down syndrome. CMAJ 1996;154:465-79.

34. Smith DK, Shaw RW, Marteau TM. Informed consent to undergo serum screening for Down's syndrome: the gap between policy and practice. BMJ 1994;309:776.

35. Goel V, Glazier R, Holzapfel S, Pugh P, Summers A. Evaluating patient's knowledge of maternal serum screening. Prenat Diagn 1996;16:425-30.

36. Green JM. Serum screening for Down's syndrome: experiences of obstetricians in England and Wales. BMJ 1994;309:769-72.

37. Sadler M. Serum screening for Down's syndrome: $\overrightarrow{\dot{\omega}}$ how much do health professionals know? Br J Obstet Gynaecol 1997;104:176-9.

38. Salonen R, Kurki L, Lappalainen M. Experiences of $\frac{0}{3}$ mothers participating in maternal serum screening for Down's syndrome. Eur J Hum Genet 1996;4: $\frac{N}{G}$ 113-9.

39. Abuelo DN, Hopmann MR, Barsel-Bowers G, Goldstein A. Anxiety in women with low maternal serum alpha-fetoprotein screening. Prenat Diagn is 1991;11:381-5.

40. Marteau TM, Cook R, Kidd J, et al. The psychological effects of false-positive results in prenatal screen- $\mathbb{\Phi}$ ing for fetal abnormality: a prospective study. Prenat Diagn 1992;12:205-14.

41. Capen K. New prenatal screening procedures raise $\square$ spectre of more "wrongful-birth" claims. CMAJ 1995;152:734-7.

42. Geller G, Tambor ES, Chase GA, Hofman KJ, Faden RR, Holtzman NA. Incorporation of genetics in primary care practice. Will physicians do the counseling and will they be directive? Arch Fam Med 1993;2:1119-25.

43. Kessler, S. Psychological aspects of genetic counseling. VII. Thoughts on directiveness. J Genet Counseling 1992;1:9-17.

44. Kessler S. Psychological aspects of genetic counseling. VIII. Suffering and countertransference. J Genet Counseling 1992;1:303-8.

45. Reif M, Baitsch H. Psychological issues in genetic counseling. Hum Genet 1985;70:193-9.

46. A blood screening test for any pregnant woman, multiple protein marker test: a fact sheet. Pacific Northwest Regional Genetics Group. Eugene, Ore: Oregon Health Sciences University, 1996.

47. Ormond KE, Pergament E, Fine BA. Pre-screening 6 education in multiple marker screening programs: the effect on patient anxiety and knowledge. J Ge- netic Counseling 1996;5:69-80.

48. Goel V, Glazier R, Holzapfel S, Pugh P, Summers A. Evaluating patient's knowledge of maternal serum $\stackrel{\square}{\perp}$ screening. Prenat Diagn 1996;16:425-30. 